\title{
Genetic impact dominates over environmental effects in development of carotid artery stiffness: a twin study
}

\author{
Tamás Horváth ${ }^{1,8}$, János Osztovits ${ }^{2,8}$, Alexandra Pintér ${ }^{1}$, Levente Littvay ${ }^{3}$, Domonkos Cseh $^{1}$, \\ Ádám D Tárnoki ${ }^{4}$, Dávid L Tárnoki ${ }^{4}$, Ádám L Jermendy ${ }^{5}$, Rita Steinbach ${ }^{5}$, Júlia Métneki ${ }^{6}$, \\ Giuseppe Schillaci ${ }^{7}$, Márk Kollai ${ }^{1}$ and György Jermendy ${ }^{2}$
}

\begin{abstract}
Arterial stiffness is an independent predictor of cardiovascular, cerebrovascular and all-cause mortality. Quantifying the genetic influence on the stiff arterial phenotype allows us to better predict the development of arterial stiffness. In this study, we aimed to determine the heritability of carotid artery stiffness in healthy twins. We studied 98 twin pairs of both sexes. We determined carotid artery stiffness locally using echo tracking and applanation tonometry. We estimated the heritability of stiffness parameters using structural equation modeling. The carotid distensibility coefficient showed the highest heritability $(64 \%, 95 \%$ confidence interval $45-77 \%$ ). The incremental elastic modulus, compliance and stiffness index $\beta$ also showed substantial heritability $(62 \%, 61 \%$ and $58 \%$, respectively). The remaining $36-42 \%$ phenotypic variance was attributed to unshared environmental effects. Genetic influence appears to dominate over environmental factors in the development of carotid artery stiffness. Environmental factors may have an important role in favorably influencing the genetic predisposition for accelerated arterial stiffening.
\end{abstract}

Hypertension Research (2014) 37, 88-93; doi:10.1038/hr.2013.133; published online 3 October 2013

Keywords: carotid artery; echo track; heritability; stiffness; twin

\section{INTRODUCTION}

The windkessel function of the central elastic arteries is important to maintain adequate perfusion of the peripheral circulation during the cardiac cycle. Owing to arterial wall stiffening with advancing age, the windkessel function of the large elastic arteries declines. In the physiological effect of aging, the additional impact of hypertension, diabetes, hypercholesterolemia and systemic inflammation can accelerate the development of arterial stiffness.

Increased central arterial stiffness leads to elevated left ventricular afterload, which ultimately leads to left ventricular hypertrophy, myocardial infarction and heart failure. ${ }^{1}$ Similarly, the incidence of stroke $^{2}$ and kidney disease ${ }^{3}$ increases along with the deterioration of windkessel function. In these pathological states, even after adjustment for known cardiovascular risk factors, arterial stiffness remains a significant predictor of cardiovascular events. ${ }^{4}$

In the present study, we investigated carotid arteries, which are representative of central elastic arteries and commonly subjected to athero- and arteriosclerotic processes. ${ }^{5}$ In addition to the hemodynamic consequences of increased arterial stiffness, the wall of the carotid artery contains high-pressure arterial baroreceptors.
Cardiac autonomic balance is strongly influenced by the arterial baroreflex, and sensitivity of the reflex is determined by stiffness of the vessel wall in which the receptors are embedded. ${ }^{6}$ Carotid artery stiffness varies considerably even in healthy individuals. The underlying mechanism for this variability has not been determined. ${ }^{7}$

Established cardiovascular risk factors can only partially explain the variability in carotid artery stiffness. Therefore, it has been proposed to extend cardiovascular risk assessment with the estimation of genetic predisposition on stiff arterial phenotype. The quantification of this hereditary risk was conducted in clinical studies on $\operatorname{twin}^{8-13}$ and family ${ }^{14-16}$ samples. Although family studies can derive the percentage of variation attributable to family sources, twin studies are more powerful because they can best separate the familial variation into genetic and environmental sources that are shared by the family. The shared familial environments are closest with twins who provide us with the most powerful tool to separate genetic similarity from familial environmental similarity. This factor is especially important with cardiovascular disease because, in addition to genetic factors, it also has been tied to lifestyle habits and socioeconomic status that could partially or completely be driven by nongenetic familial sources. ${ }^{17}$

${ }^{1}$ Institute of Human Physiology and Clinical Experimental Research, Semmelweis University, Budapest, Hungary; ${ }^{2}$ Medical Department, Bajcsy-Zsilinszky Hospital, Budapest, Hungary; ${ }^{3}$ Central European University, Budapest, Hungary; ${ }^{4}$ Department of Radiology and Oncotherapy, Semmelweis University, Budapest, Hungary; ${ }^{5}$ Semmelweis University, Faculty of Medicine, Budapest, Hungary; ${ }^{6}$ National Centre for Healthcare Audit and Inspection, Budapest, Hungary and ${ }^{7}$ Department of Medicine, University of Perugia, and Unit of Internal Medicine, Terni University Hospital, Terni, Italy

8These authors contributed equally to this work.

Correspondence: Professor G Schillaci, Department of Medicine, University of Perugia, and Unit of Internal Medicine, Terni University Hospital, Viale Tristano di Joannuccio, 1-05100 Terni, Italy. 
Monozygotic (MZ) twins share 100\% of their genome, whereas dizygotic (DZ) twins share half of their genes on average. Moreover, $\mathrm{MZ}$ and DZ twins share their intrauterine life, having the same age and early extrauterine environment. On a population level, these features allow disease-associated phenotypic variations to be most accurately broken down to shared genetic, shared environmental and unique environmental components. By quantifying the variation of these components as a part of a complex variation of a trait, heritability estimates can be made. Heritability can be defined on a population-based level, given as the variation of the phenotype explained by the variation of the common genetic component. ${ }^{18}$

The aim of this study was to determine the relative contribution of genetic and environmental effects on carotid artery stiffness. We performed a classical twin study to investigate adult MZ and DZ twin pairs by using both carotid ultrasonography and tonometry.

\section{METHODS}

In the present study, we investigated 98 adult twin pairs $(n=196$; women $71 \%$; mean age 43 years, range 18-81 years). We recruited the participants from the recently established Hungarian twin registry, ${ }^{19}$ from twin meetings and through advertisements. Because of the lack of genotyping data for the subjects, we used a multiple self-reported question approach to assess zygosity in order to maximize the accuracy of classification. The most likely zygosity was assigned based on the seven self-reported responses. ${ }^{20}$ We investigated 61 $\mathrm{MZ}$ and $37 \mathrm{DZ}$ twin pairs of the same gender. Exclusion criteria included pregnancy, diabetes mellitus, myocardial infarction or regular alcohol consumption (more than 2 units daily) in the recent medical history, conditions likely to interfere with compliance during test procedures and acute infection within 3 weeks of measurement.

We performed anthropometric measurements (recording weight, height and waist circumference) and a complete physical examination. We calculated body mass index from the values of weight and height. We measured waist circumference using the standard method. We assessed physical activity level using the following standardized method: the subjects reported the amount of time spent on five different intensity levels of physical activity on an average weekday as a total of $24 \mathrm{~h}$; subsequently, the values of daily metabolic equivalent scores were derived and used for statistical analysis. ${ }^{21}$ Alcohol consumption was assessed as units per week and coffee consumption as cups per day. For the statistical analyses related to the participant's alcohol consumption and smoking habits, we split our sample into drinkers and nondrinkers and smokers and nonsmokers. Fasting venous blood samples were obtained from twin pairs and routine laboratory methods were used for measuring blood glucose, lipids and serum creatinine.

Anthropometric data and medical history, along with lifestyle and zygosity questionnaires were obtained and evaluated by one group of researchers at one site (Bajcsy-Zsilinszky Hospital), whereas the carotid examinations were conducted by another group of operators at a different site (Institute of Human Physiology). The carotid examiners were blinded to zygosity, laboratory data and medical history of the participants.

All of the participants provided their written informed consent. The investigation was approved by the National Research Ethics Committee (ETT TUKEB, Budapest) and was conducted according to the principles expressed in the Declaration of Helsinki.

\section{Determination of carotid artery stiffness}

Local arterial stiffness of the left common carotid artery was assessed using ultrasonography and applanation tonometry. The investigations were performed in the early afternoon under standardized conditions. All of the individuals refrained from smoking and fasted at least $12 \mathrm{~h}$ before testing. Additionally, all of the subjects were asked to abstain from strenuous physical activity or drinking alcohol or caffeinated beverages for $24 \mathrm{~h}$ before the investigation. After arrival, the subjects were equipped with the appropriate devices, and then rested in a supine position for $\sim 15 \mathrm{~min}$ until baseline conditions for heart rate and mean blood pressure were reached.

\section{Ultrasonography}

Outer end-diastolic diameter (Dd), intima-media thickness (IMT) and pulsatile distension of the common carotid artery were determined by echo tracking. A minimum $1.5-\mathrm{cm}$ segment of the common carotid artery was insonated $\sim 2 \mathrm{~cm}$ proximal to the bifurcation with a 7.5 - to $10-\mathrm{MHz}$ linear scanner (L10-5, $40 \mathrm{~mm}$, Picus Pro, Esaote, The Netherlands). The radiofrequency signal of the ultrasound device was analyzed using Art.Lab (Art.Lab, Esaote, The Netherlands). Depending on the operational mode of the system, a 6-s-long measurement session of the insonated vessel segment consisted of up to 23040 parallel measurements of vascular geometry (diameter, IMT). For each participant, the results of the three sessions were averaged and documented. Additional descriptions of the method and repeatability data were published previously. ${ }^{22}$ Based on the outer diameter and IMT data, the lumen cross-sectional area (Lcsa) and the intima-media cross-sectional area (IMcsa) were calculated using the formulae: Lcsa $=\pi \times(\mathrm{Dd} / 2-\mathrm{IMT})^{2}$ and $\mathrm{IMcsa}=\pi \times(\mathrm{Dd} / 2)^{2}-$ Lcsa.

\section{Carotid artery pulse wave analysis}

Carotid artery blood pressure was measured using applanation tonometry (SPT-301, Millar Instruments, Houston, TX, USA). Calibration of the tonometric signal was based on the average of a minimum of three resting, supine sphygmomanometric (Colin CBM-7000) readings from the right arm. Tonometric data were recorded and analyzed using SphygmoCor software, version 7.0 (AtCor, Sydney, NSW, Australia), and carotid systolic, diastolic and pulse pressure values were determined.

\section{Indices of carotid stiffness}

Measurements of the carotid diameter and pressure and the consequent calculations of vascular stiffness parameters were performed according to international guidelines. ${ }^{23,24}$ The carotid artery compliance and distensibility were expressed as compliance and distensibility coefficients and defined as the absolute and relative changes in the cross-sectional area of the vessel per unit of pressure change. The incremental elastic modulus was calculated as $[3 \times(1+\mathrm{Lcsa} / \mathrm{IMcsa})] /$ DC. The stiffness index $\beta$ was determined by the formula: $\ln (\mathrm{SBPc} / \mathrm{DBPc}) /(\mathrm{Dist} / \mathrm{Dd})$.

\section{Statistical analysis}

Sample assessment. Assessing similarities and differences between the MZ and DZ subsamples is important to understand the likely sources of bias in the results. We used a parametric difference test with cluster-sampling correction. ${ }^{25}$ The correction was needed because the observations in the sample were not completely independent of each other. The correction accounted for the difference in trait variance within and between clusters (families). Parametric tests were sufficient because all raw or log-transformed continuous traits were within acceptable parameters of normality, and nonparametric tests do not offer such clustering corrections. Values represented the mean \pm s.d. The differences between parametric tests were considered to be significant at $P<0.05$. For dichotomous predictors, the differences in proportions were presented and the hypothesis test was performed using a log-link function. ${ }^{26}$

Risk factors. To better understand the role of risk factors, age- and gendercorrected bivariate correlations were derived between the dependent variables and the risk factors. Bivariate correlations also utilized cluster-sampling standard errors for correct hypothesis tests. Bivariate relationships were considered to be significant at $P<0.05$.

The parameters failed to show a normal distribution for MZ-DZ comparisons and for the correlation analyses were log-normalized.

Genetic and environmental impact. Co-twin correlations for arterial stiffness parameters were determined using two models (model-1 and model-2). Model-1 corrects for the twins' age and gender. Model-2, in addition to age and gender, corrects for all of the significant risk factors based on the bivariate correlations. A substantially higher MZ co-twin correlation (compared to DZ correlations) suggests heritability, whereas similar co-twin correlations imply 
that shared environmental components drive the variance more strongly. The deviation from perfect $\mathrm{MZ}$ co-twin correlations signifies that a portion of the variance is unrelated to genetic and shared environmental effects.

In addition to correlation-based similarities between $\mathrm{MZ}$ and $\mathrm{DZ}$ twins, a structural equation model, often called the A-C-E model, was constructed. In the A-C-E model, three latent variables, additive genetic effects ' $\mathrm{A}$ ', common (or shared) environment ' $C$ ' and unshared (or unique) environment ' $E$ ', drive the variance in the phenotype for each twin. ${ }^{27} \mathrm{~A}$ is perfectly (1.0) correlated across MZ twins and 0.5 correlated across $\mathrm{DZ}$ twins. C is perfectly correlated independently of zygosity. $\mathrm{E}$ is uncorrelated across co-twins. Because the measurement error in the phenotype is also uncorrelated across measurements, it appears as part of the unique environmental (E) component. Considering the well-established reliable measures used in this study, this property of the model is of little concern. This A-C-E structural equation model is graphically represented in Figure 1.

Two A-C-E models (model-1 and model-2 as used for co-twin correlations) were estimated. The correction for age and gender is justified by gender being $100 \%$ genetic and age being $100 \%$ environmental. All other predictors could carry both a genetic and an environmental component. The results from model- 1 tell us the total genetic and environmental impact on the dependent variable. The results from model-2 tell us the impact of genes and the environment after the impact of known risks is corrected for. Empirically derived bootstrapped confidence intervals are presented for the heritability and environmental proportion estimates. ${ }^{28}$ Standard hypothesis tests are inappropriate for these because the proportions are bounded and can never be negative; therefore, the central limit theorem assumptions consisting of traditional standard error-based hypothesis tests are inappropriate. All of the inferential statistics were estimated using full information maximum likelihood and Mplus version 6 software. ${ }^{29}$

\section{RESULTS}

The zygosity-specific demographic characteristics and the distribution of cardiovascular risk factors, as well as arterial hemodynamic indices, are presented in Table 1 . All of the displayed parameters showed agerelated normal values. Approximately $30 \%$ of our subjects were smokers and approximately $40 \%$ consumed alcohol; however, their consumption habits did not reach the exclusion criteria of 2 units/day.

The correlations of hemodynamic indices with anthropometric parameters and cardiovascular risk factors are shown in Table 2. Covariates were entered for structural equation modeling (model-2) that reached $P<0.1$. This unconventional level of certainty was selected to ensure that we do not miss any important covariates.

Co-twin correlations within model- 1 and model- 2 are shown in Table 3. Upon assessment, we observed that most phenotypes proved

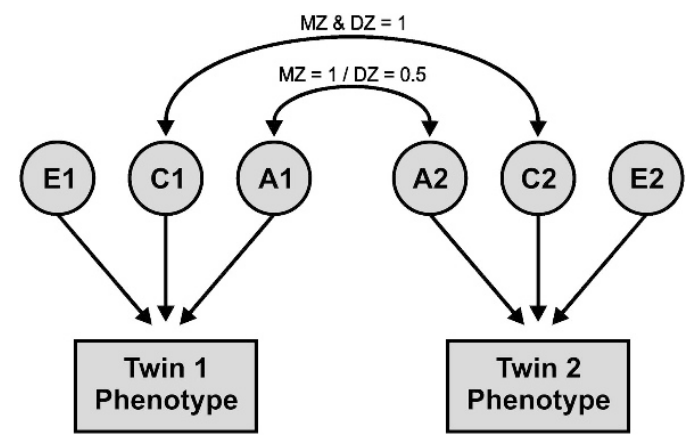

Figure 1 Univariate A-C-E model. Rectangles denote the observed variables (stiffness) and circles denote the latent variables. Curved arrows denote correlations (fixed at the highlighted values). Straight arrows signify the estimated impact of the latent factor on the variance of the observed phenotype. Letters A, C, E represent additive genetic, common environmental and unique environmental influences, respectively. to be highly heritable. Comparing model-1 with model-2, we found that the magnitudes of $\mathrm{MZ}$ and $\mathrm{DZ}$ co-twin correlations were not substantially impacted by the correction of relevant covariates.

Using the most parsimonious (the A-E models for all parameters) structural equation model (Table 4 and Figure 2), the heritability (genetic influence) of different hemodynamic parameters was estimated between 34 and $64 \%$ in our sample. Common (shared) environmental effects did not exhibit a significant influence on the dependent parameters, whereas unshared (unique) environmental influence was observed between 36 and $66 \%$ in the crude model (model-1) where hemodynamic indices were adjusted for age and gender only.

\section{Table 1 Characteristics of MZ and DZ twins}

\begin{tabular}{lccc}
\hline Parameter & $M Z(\mathrm{n}=122)$ & $D Z(\mathrm{n}=74)$ & P-value \\
\hline Anthropometric & & & \\
Women (\%) & 72 & 70 & 0.844 \\
Age (years) & $47 \pm 15$ & $37 \pm 13$ & 0.001 \\
Body mass index (kg m ${ }^{-2}$ ) & $26 \pm 5$ & $25 \pm 6$ & 0.597 \\
Waist circumference (cm) & $88 \pm 15$ & $87 \pm 16$ & 0.738 \\
Heart rate (beats per min) & $67 \pm 10$ & $68 \pm 7$ & 0.248 \\
Systolic blood pressure (mmHg) & $131 \pm 15$ & $125 \pm 14$ & 0.028 \\
Diastolic blood pressure (mmHg) & $75 \pm 10$ & $72 \pm 10$ & 0.177 \\
Pulse pressure (mmHg) & $56 \pm 9$ & $53 \pm 8$ & 0.041 \\
& & & \\
Central arterial hemodynamic & & & \\
Systolic blood pressure (mmHg) & $120 \pm 15$ & $114 \pm 13$ & 0.017 \\
Diastolic blood pressure (mmHg) & $75 \pm 10$ & $72 \pm 10$ & 0.179 \\
Pulse pressure (mmHg) & $44 \pm 8$ & $41 \pm 8$ & 0.041
\end{tabular}

\section{Laboratory}

Fasting blood glucose $\left(\mathrm{mmoll}^{-1}\right)$

Serum fasting insulin $\left(\mu \cup \mathrm{ml}^{-1}\right)^{\mathrm{a}}$

$4.9 \pm 0.6$

$4.8 \pm 0.5$

0.118

Serum total cholesterol $\left(\mathrm{mmol}^{-1}\right)$

$7.21 \pm 4.99$

$6.85 \pm 4.58$

0.789

Serum HDL-cholesterol (mmol ${ }^{-1}$ )

$5.34 \pm 1.23$

$4.86 \pm 1.08$

Serum triglycerides $\left(\mathrm{mmoll}^{-1}\right)^{\mathrm{a}}$

Serum creatinine $\left(\mu \mathrm{moll}^{-1}\right.$ )

$1.58 \pm 0.35$

$1.57 \pm 0.28$

$1.34 \pm 0.86$

$1.02 \pm 0.78$

$73 \pm 11$

Serum C-reactive protein $\left(\mathrm{mg} \mathrm{I}^{-1}\right)^{\mathrm{a}}$

Serum uric acid ( $\mu \mathrm{moll}^{-1}$ )

$70 \pm 10$

$2.96 \pm 1.86$

$3.75 \pm 3.65$

$289 \pm 75$

$279 \pm 72$

Serum homocystein $\left(\mu \mathrm{moll} \mathrm{I}^{-1}\right.$ )

$11.4 \pm 3.6$

$11.2 \pm 3.2$

0.027

0.880

0.004

0.219

0.565

0.464

0.718

\section{Lifestyle}

Physical activity (MET per day)

Current smokers (\%)

Alcohol consumers (\%)

Coffee consumers (\%)

Coffee consumption $>1$ per day (\%)

$65.8 \pm 22.1$
32.2
44.3
73.0
49.2

$59.6 \pm 20.3$
31.1
43.2
55.4
31.1

0.122

0.888

0.913

0.044

0.028

$\begin{array}{ccr}6.18 \pm 0.73 & 5.97 \pm 0.82 & 0.126 \\ 568 \pm 126 & 479 \pm 119 & <0.001 \\ 0.10 \pm 0.03 & 0.11 \pm 0.03 & 0.091 \\ 3.11 \pm 1.44 & 3.64 \pm 1.38 & 0.051 \\ 8.69 \pm 3.93 & 7.28 \pm 2.62 & 0.045 \\ 4.01 \pm 2.17 & 3.63 \pm 1.80 & 0.427\end{array}$

bbreviations: CC, compliance; DC, distensibility; DZ, dizygotic; MZ, monozygotic. Data are presented as means (or percentages) \pm s.d. (standard deviations). IMT stands for the intima - media thickness of the common carotid artery. CC and DC coefficients are measures of vascular elasticity, whereas stiffness index $\beta$ and incremental elastic modulus (termed as Einc) are measures of vascular stiffness. For quantification of elastic modulus (termed as Einc) are measures of vascular stiffness. For quantification
physical activity, the unit of metabolic equivalent of task (termed as MET) was used. aparameters were log-normalized before statistical analyses. 
Table 2 Association of carotid artery parameters with traditional risk factors

\begin{tabular}{lcccc}
\hline Covariate & $C C$ & $D C$ & Stiffness index $\beta$ & Einc \\
\hline Body mass index & $-0.22^{\mathrm{a}}$ & $-0.36^{\mathrm{b}}$ & $0.30^{\mathrm{b}}$ & $0.41^{\mathrm{b}}$ \\
Waist circumference & $-0.25^{\mathrm{a}}$ & $-0.38^{\mathrm{b}}$ & $0.31^{\mathrm{b}}$ & $0.40^{\mathrm{b}}$ \\
Heart rate & -0.03 & -0.06 & 0.01 & 0.02 \\
Systolic blood pressure (brachial) & $-0.23^{\mathrm{b}}$ & $-0.35^{\mathrm{b}}$ & $0.12^{\mathrm{c}}$ & $0.44^{\mathrm{b}}$ \\
Diastolic blood pressure (brachial) & $-0.15^{\mathrm{a}}$ & $-0.27^{\mathrm{b}}$ & -0.01 & $0.30^{\mathrm{a}}$ \\
Pulse pressure (brachial) & $-0.18^{\mathrm{a}}$ & $-0.22^{\mathrm{b}}$ & $0.18^{\mathrm{a}}$ & $0.31^{\mathrm{b}}$ \\
Systolic blood pressure (carotid) & $-0.27^{\mathrm{b}}$ & $-0.38^{\mathrm{b}}$ & $0.15^{\mathrm{a}}$ & $0.49^{\mathrm{b}}$ \\
Diastolic blood pressure (carotid) & $-0.15^{\mathrm{a}}$ & $-0.27^{\mathrm{b}}$ & 0.00 & $0.30^{\mathrm{a}}$ \\
Pulse pressure (carotid) & $-0.21^{\mathrm{b}}$ & $-0.23^{\mathrm{b}}$ & $0.19^{\mathrm{a}}$ & $0.30^{\mathrm{b}}$ \\
Serum cholesterol & $-0.15^{\mathrm{a}}$ & $-0.14^{\mathrm{c}}$ & 0.10 & $0.20^{\mathrm{a}}$ \\
Serum triglycerides & $-0.12^{\mathrm{c}}$ & $-0.21^{\mathrm{b}}$ & $0.16^{\mathrm{a}}$ & $0.23^{\mathrm{a}}$ \\
Serum HDL-cholesterol & $-0.17^{\mathrm{a}}$ & -0.10 & $0.14^{\mathrm{c}}$ & 0.05 \\
Serum creatinine & 0.06 & 0.05 & 0.00 & 0.01 \\
Serum uric acid & -0.09 & $-0.18^{\mathrm{a}}$ & $0.18^{\mathrm{a}}$ & $0.24^{\mathrm{b}}$ \\
Serum C-reactive protein & -0.03 & $-0.16^{\mathrm{a}}$ & 0.08 & $0.20^{\mathrm{a}}$ \\
Serum homocystein & 0.01 & 0.00 & -0.05 & 0.04 \\
Serum glucose & -0.07 & -0.09 & $0.12^{\mathrm{c}}$ & $0.16^{\mathrm{a}}$ \\
Serum insulin & -0.06 & $-0.15^{\mathrm{a}}$ & $0.15^{\mathrm{a}}$ & $0.24^{\mathrm{b}}$ \\
Physical activity & -0.03 & -0.02 & 0.02 & -0.01 \\
Alcohol consumption & 0.08 & 0.02 & -0.08 & -0.06 \\
Coffee consumption & -0.08 & $-0.15^{\mathrm{a}}$ & 0.09 & 0.04 \\
Smoking habits & -0.02 & -0.07 & 0.06 & 0.06 \\
\hline
\end{tabular}

Abbreviations: CC and DC, compliance and distensibility coefficients; Einc, incremental elastic modulus; IMT, carotid intima - media thickness.

Cluster-corrected standardized correlation coefficients between variables.

bCorrelations are significant at level $P<0.001$

'Statistical tendency at level $P<0.1$.

Table 3 Corrected, bivariate, co-twin correlations for twins (monozygotic (MZ), $n=61$; dizygotic (DZ), $n=37$ twins)

\begin{tabular}{lcccc}
\hline Dependent variables & $M Z$ correlation & $95 \% \mathrm{Cl}$ & DZ correlation & $95 \% \mathrm{Cl}$ \\
\hline CC & & & & \\
Model 1 & 0.60 & $0.39-0.75$ & 0.37 & $0.12-0.59$ \\
Model 2 & 0.58 & $0.35-0.75$ & $\mathbf{0 . 3 9}$ & $0.13-0.67$ \\
DC & & & & \\
Model 1 & 0.64 & $0.45-0.77$ & $\mathbf{0 . 3 3}$ & $0.01-0.60$ \\
Model 2 & $\mathbf{0 . 5 8}$ & $0.33-0.78$ & $\mathbf{0 . 3 9}$ & $0.07-0.71$ \\
& & & & \\
Stiffness $\beta$ & & & & \\
Model 1 & $\mathbf{0 . 5 8}$ & $0.29-0.77$ & $\mathbf{0 . 1 9}$ & $-0.17-0.53$ \\
Model 2 & $\mathbf{0 . 5 2}$ & $0.23-0.77$ & $\mathbf{0 . 1 5}$ & $-0.45-0.69$ \\
& & & & \\
Einc & & & & \\
Model 1 & $\mathbf{0 . 6 4}$ & $0.47-0.77$ & $\mathbf{0 . 1 1}$ & $-0.32-0.53$ \\
Model 2 & $\mathbf{0 . 6 0}$ & $0.33-0.78$ & $\mathbf{0 . 0 9}$ & $-0.40-0.57$ \\
\hline
\end{tabular}

Abbreviations: CC and DC, compliance and distensibility coefficients; Einc, incremental elastic modulus; IMT, carotid intima - media thickness.

Model 1 -correlations were adjusted to age and sex.

Model 2-correlations were adjusted to age, sex and all associated covariates (see Table 2).

\section{DISCUSSION}

Our classical twin study documented that genetic factors exert a substantial influence on carotid artery stiffness parameters. We evaluated various indices of carotid elasticity and stiffness, reflecting different aspects of noninvasive vascular stiffness. We used high- resolution ultrasonographic vessel wall tracking together with applanation tonometry to accurately derive these values. According to the recent consensus document on arterial stiffness determination, echo tracking together with local pulse pressure determination is the preferred method for determining local arterial stiffness. ${ }^{23}$ Our data demonstrated that the variances of carotid stiffness parameters were driven approximately $60 \%$ by additive genetic effects, whereas the remaining portion of the variances consisted of unique environmental factors.

In the setting of the Northern Manhattan Family Study, the heritability of carotid distensibility, stiffness and elastic modulus was found to be substantially lower than our results (17\%, 20\% and $20 \%$, respectively). ${ }^{14}$ Although the methods for assessing carotid distensibility were similar in both studies, it should be noted that (i) the study was conducted on families and not on twins, (ii) the sample populations were of different ethnicity, and (iii) the Northern Manhattan Family Study participants were at higher cardiovascular risk. The Northern Manhattan Family Study determined carotid geometry using a video-based approach. According to the consensus study on arterial stiffness, a pixel-based determination might introduce an $\sim 150-\mu \mathrm{m} /$ pixel measurement error in contrast to wall tracking, which reports an error of $\sim 1 \mu \mathrm{m} .^{23}$ That larger measurement error inflates unique environmental estimates that might also explain the different results between our studies.

Although our study shows a strong genetic predisposition to the stiff carotid arterial phenotype, it is worth emphasizing that the remaining $40 \%$ of carotid stiffness is driven by unique environmental factors, which may indicate the importance of increased attention to healthy lifestyle measures that have the potential to prevent and reduce the age-dependent increase of arterial stiffness, such as sodium restriction, consumption of omega- 3 fatty acids, ${ }^{30}$ smoking cessation, ${ }^{31}$ and regular physical activity. ${ }^{32}$

\section{Limitations}

Our twin sample size was modest, although comparable to other clinical studies with twins. Because of our lack of genetic data, the zygosity in our twin cohort was classified according to validated questionnaires. Nevertheless, this method is widely accepted in clinically oriented twin studies. ${ }^{20}$

Anthropometric and central arterial hemodynamic parameters within our sample demonstrated that MZ twins are significantly older (on average by 10 years) and have higher values of brachial and carotid systolic (by $6 \mathrm{~mm} \mathrm{Hg}$ on average) and pulse pressures (by $3 \mathrm{~mm} \mathrm{Hg}$ on average). As for the traditional cardiovascular risk factors, the serum total cholesterol and triglyceride values were higher in MZ versus DZ twin pairs. These significant differences are likely solely the function of age. Our data were corrected for age and gender (model-1) and for other significant covariates in estimation (model-2).

Although diabetes mellitus was considered to be an exclusion criterion, we investigated only fasting blood glucose values and we did not conduct an oral glucose tolerance test in our cohort. Despite compliance, the distensibility and incremental elastic moduli of MZ and DZ twins did not differ substantially; the stiffness index $\beta$ of MZ twins showed a higher value.

In our model, we considered alcohol, coffee, and tobacco use to be unique environmental (E) factors. Although this is a simplification we decided to make, alcohol and smoking habits along with coffee consumption might carry genetic components because it is known that smoking (and even propensity to avoid cigarette smoke) is driven by additive genetic factors. Although smoking and drinking habits as 
Table 4 Parameter estimates for additive genetics (A), common environment (C) and unique environmental influences (E) on carotid stiffness parameters by structural equation modeling

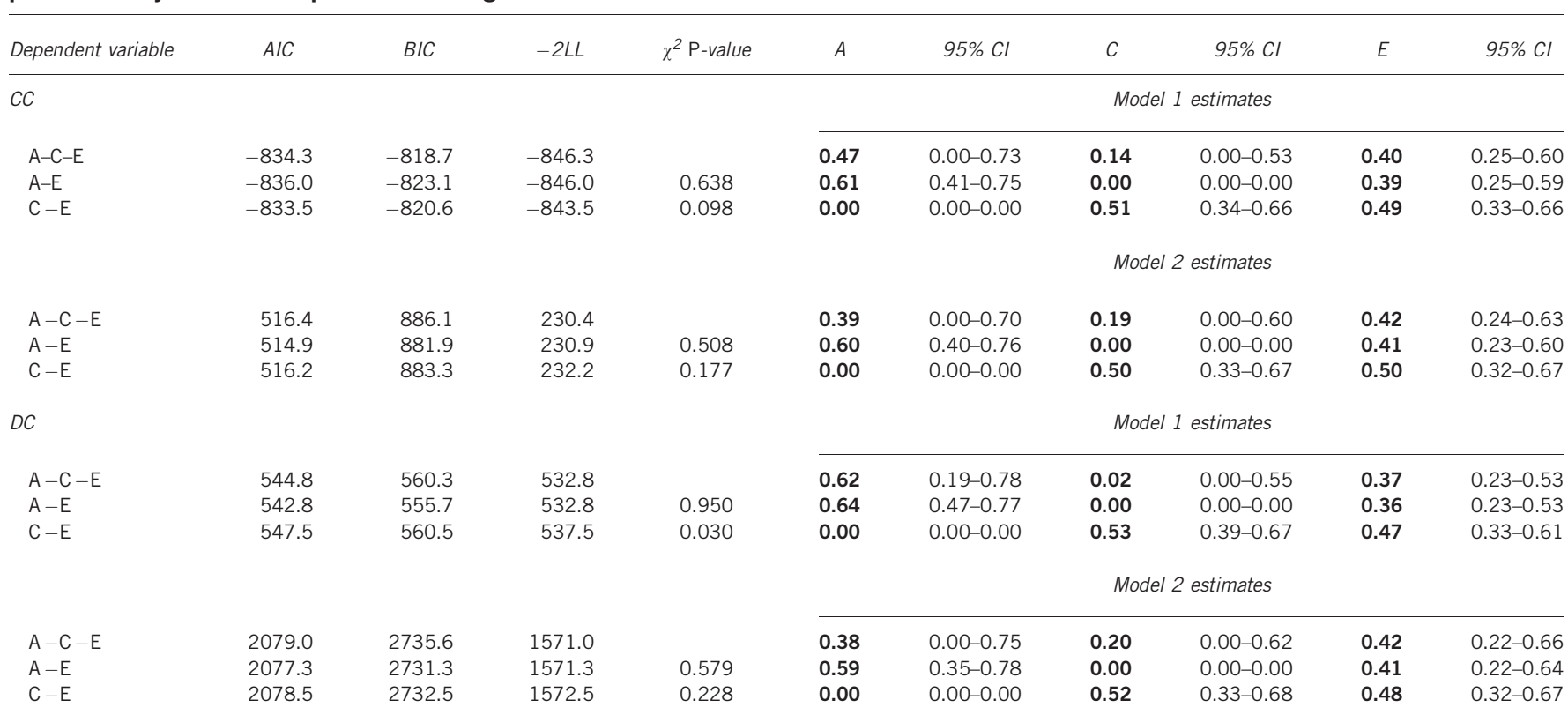

Stiffness $\beta$

$$
\begin{aligned}
& A-C-E \\
& A-E \\
& C-E
\end{aligned}
$$

53.6
51.6
56.0

$$
\begin{aligned}
& 69.1 \\
& 64.5 \\
& 68.9
\end{aligned}
$$



$A-C-E$

A - E

C $-\mathrm{E}$

Einc

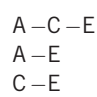

$A-C-E$
$A-E$

$C-E$
159.2

157.2
166.0

$$
\begin{aligned}
& 174.7 \\
& 170.2
\end{aligned}
$$

170.2
179.0

$$
\begin{aligned}
& 41.6 \\
& 41.6 \\
& 46.0
\end{aligned}
$$

\begin{tabular}{|c|c|c|c|c|c|c|c|c|c|c|}
\hline$A-C-E$ & 1954.8 & 2782.0 & 1314.8 & & 0.59 & $0.03-0.80$ & 0.00 & $0.00-0.57$ & 0.41 & $0.21-0.71$ \\
\hline$A-E$ & 1952.8 & 2777.4 & 1314.8 & 1.000 & 0.59 & $0.30-0.78$ & 0.00 & $0.00-0.00$ & 0.41 & $0.22-0.69$ \\
\hline$C-E$ & 1959.7 & 2784.3 & 1321.7 & 0.008 & 0.00 & $0.00-0.00$ & 0.43 & $0.20-0.63$ & 0.57 & $0.36-0.80$ \\
\hline
\end{tabular}

Model 1 estimates

\begin{tabular}{llllll}
\hline $\mathbf{0 . 5 8}$ & $0.02-0.79$ & $\mathbf{0 . 0 0}$ & $0.00-0.00$ & $\mathbf{0 . 4 2}$ & $0.23-0.70$ \\
$\mathbf{0 . 5 8}$ & $0.30-0.77$ & $\mathbf{0 . 0 0}$ & $0.00-0.00$ & $\mathbf{0 . 4 2}$ & $0.23-0.69$ \\
$\mathbf{0 . 0 0}$ & $0.00-0.00$ & $\mathbf{0 . 4 9}$ & $0.30-0.66$ & $\mathbf{0 . 5 1}$ & $0.34-0.70$
\end{tabular}

Model 2 estimates

\begin{tabular}{llllll}
\hline $\mathbf{0 . 5 1}$ & $0.00-0.76$ & $\mathbf{0 . 0 0}$ & $0.00-0.65$ & $\mathbf{0 . 4 9}$ & $0.24-0.77$ \\
$\mathbf{0 . 5 1}$ & $0.25-0.77$ & $\mathbf{0 . 0 0}$ & $0.00-0.00$ & $\mathbf{0 . 4 9}$ & $0.23-0.75$ \\
$\mathbf{0 . 0 0}$ & $0.00-0.00$ & $\mathbf{0 . 4 5}$ & $0.24-0.66$ & $\mathbf{0 . 5 5}$ & $0.34-0.76$
\end{tabular}

Model 1 estimates

$\begin{array}{ll}147.2 & \\ 147.2 & 1.000 \\ 156.0 & 0.003\end{array}$

\begin{tabular}{llllll}
\hline $\mathbf{0 . 6 2}$ & $0.37-0.78$ & $\mathbf{0 . 0 0}$ & $0.00-0.58$ & $\mathbf{0 . 3 8}$ & $0.23-0.54$ \\
$\mathbf{0 . 6 2}$ & $0.46-0.77$ & $\mathbf{0 . 0 0}$ & $0.00-0.00$ & $\mathbf{0 . 3 8}$ & $0.23-0.54$ \\
$\mathbf{0 . 0 0}$ & $0.00-0.00$ & $\mathbf{0 . 4 7}$ & $0.27-0.64$ & $\mathbf{0 . 5 3}$ & $0.36-0.73$
\end{tabular}

Model 2 estimates

Abbreviations: AIC, Akaike information criteria; BIC, Bayesian information criteria; CC and DC, compliance and distensibility coefficients; Einc, incremental elastic modulus; LL, log likelihood; $\chi^{2}, \chi$-test $P$-value, based on model loglikelihood comparative model fit test; IMT, carotid intima-media thickness.

well as daily physical activity of the MZ and DZ twins appear to be identical, a higher percentage of DZ twins drank more than one cup of coffee on a daily basis. A more detailed analysis of different smoking or lifestyle habits between twin pairs would allow us to obtain more precise control over the smoking or lifestyle-induced environmental effects. However, the modest sample size prevented us from fragmenting our cohort into additional subgroups.

For model-1, we can deduce the best fitting models with statistical confidence. In looking at the results, we observe very little difference between model-1 and model-2; hence, we can conclude that the heritability proportions of the phenotypes under study are not exclusively (or even substantively) driven by the heritability of the covariates. Although the best-fitting model was always the same for model- 1 and model- 2 results based on AIC and BIC, for model-2, our ability to significantly distinguish between the best-fitting models using the chi-square nested model comparison was limited by the lower power of the models with many covariates. The impact of covariates on the heritability proportions is trivial, at best. For this reason, model-1 results remain telling.

Bearing in mind the limitations, the strengths of our study should also be noted. To evaluate carotid stiffness, we evaluated a broad range of indices. The same investigators (TH and AP) performed all of the noninvasive hemodynamic measurements using the same equipment. To the best of our knowledge, this report is the first classical twin study to document that carotid stiffness is primarily related to genetic factors using both ultrasonography and applanation tonometry. 


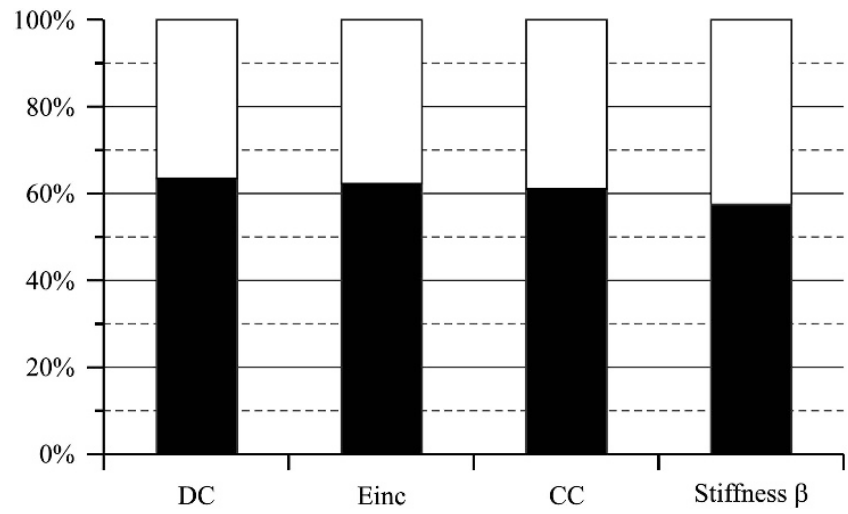

Figure 2 Age- and sex-corrected A-C-E parameter estimates based on model 1. The most parsimonious $(A-E)$, univariate structural equation model estimates for arterial stiffness parameters. Black portions indicate genetic effects (A), white portions indicate unique environmental effects $(E)$.

\section{CONFLICT OF INTEREST}

The authors declare no conflict of interest.

\section{ACKNOWLEDGEMENTS}

We thank Mrs Beáta Nagy for her valuable assistance during the examinations and Mr Gyula Németh for his excellent technical support. The study was supported by an unrestricted grant from Sanofi-Aventis. Grants from the Hungarian Diabetes Association and the Hungarian Society of Hypertension are acknowledged.

1 Mitchell GF, Hwang S-JJ, Vasan RS, Larson MG, Pencina MJ, Hamburg NM, Vita JA, Levy D, Benjamin EJ. Arterial stiffness and cardiovascular events: the Framingham Heart Study. Circulation 2010; 121: 505-511.

2 Laurent S, Katsahian S, Fassot C, Tropeano A-I, Gautier I, Laloux B, Boutouyrie P. Aortic stiffness is an independent predictor of fatal stroke in essential hypertension. Stroke 2003; 34: 1203-1206.

3 Blacher J, Guerin AP, Pannier B, Marchais SJ, Safar ME, London GM. Impact of aortic stiffness on survival in end-stage renal disease. Circulation 1999; 99: 2434-2439.

4 Laurent S. Structural and genetic bases of arterial stiffness. Hypertension 2005; 45 1050-1055.

5 Safar ME, Blacher J, Mourad JJ, London GM. Stiffness of carotid artery wall material and blood pressure in humans: application to antihypertensive therapy and stroke prevention. Stroke 2000; 31: 782-790.

6 Bonyhay I, Jokkel G, Kollai M. Relation between baroreflex sensitivity and carotid artery elasticity in healthy humans. Am J Physiol 1996; 271: H1139-H1144.

7 O'Rourke MF, Staessen JA, Vlachopoulos C, Duprez D, Plante GE. Clinical applications of arterial stiffness; definitions and reference values. Am J Hypertens 2002; 15: 426-444.

8 Cecelja M, Jiang B, McNeill K, Kato B, Ritter J, Spector TD, Chowienczyk P. Increased wave reflection rather than central arterial stiffness is the main determinant of raised pulse pressure in women and relates to mismatch in arterial dimensions. J Am Coll Cardiol 2009; 54: 695-703.
9 Jartti L, Rönnemaa T, Kaprio J, Järvisalo MJ, Toikka JO, Marniemi J, Hammar N, Alfredsson L, Saraste M, Hartiala J, Koskenvuo M, Raitakari OT. Population-based twin study of the effects of migration from Finland to Sweden on endothelial function and intima-media thickness. Arterioscler Thromb Vasc Biol 2002; 22: 832-837.

10 Snieder H, Hayward CS, Perks U, Kelly RP, Kelly PJ, Spector TD. Heritability of central systolic pressure augmentation: a twin study. Hypertension 2000; 35: 574-579.

11 Swan L, Birnie DH, Inglis G, Connell JMC, Hillis WS. The determination of carotid intima medial thickness in adults-a population-based twin study. Atherosclerosis 2003; 166: 137-141.

12 Tarnoki AD, Tarnoki DL, Stazi MA, Medda E, Cotichini R, Nisticò L, Fagnani C, Lucatelli P, Boatta E, Zini C, Fanelli F, Baracchini C, Meneghetti G, Osztovits J, Jermendy G, Preda I, Kiss RG, Metneki J, Horvath T, Karlinger K, Racz A, Lannert A, Molnar AA, Littvay L, Garami Z, Berczi V, Schillaci G. Heritability of central blood pressure and arterial stiffness: a twin study. J Hypertens 2012; 30: 1564-1571.

13 Zhao J, Cheema FA, Bremner JD, Goldberg J, Su S, Snieder H, Maisano C, Jones L, Javed F, Murrah N, Le N-A, Vaccarino V. Heritability of carotid intima-media thickness: a twin study. Atherosclerosis 2008; 197: 814-820.

14 Juo S-HH. Heritability of carotid artery distensibility in Hispanics: the Northern Manhattan Family Study. Stroke 2005; 36: 2357-2361.

15 North KE, MacCluer JW, Devereux RB, Howard BV, Welty TK, Best LG, Lee ET, Fabsitz RR, Roman MJ. Heritability of carotid artery structure and function: the Strong Heart Family Study. Arterioscler Thromb Vasc Biol 2002; 22: 1698-1703.

16 Sayed-Tabatabaei FA. Heritability of the function and structure of the arterial wall: findings of the Erasmus Rucphen Family (ERF) Study. Stroke 2005; 36: 2351-2356.

17 Jermendy G, Horvath T, Littvay L, Steinbach R, Jermendy AL, Tarnoki AD, Tarnoki DL, Metneki J, Osztovits J. Effect of genetic and environmental influences on cardiometabolic risk factors: a twin study. Cardiovasc Diabetol 2011; 10: 96.

18 MacGregor AJ, Snieder H, Schork NJ, Spector TD. Twins. Novel uses to study complex traits and genetic diseases. Trends Genet 2000; 16: 131-134.

19 Littvay L, Metneki J, Tarnoki AD, Tarnoki DL. The Hungarian Twin Registry. Twin Res Hum Genet 2013; 16: 185-189.

20 Heath AC, Nyholt DR, Neuman R, Madden PAF, Bucholz KK, Todd RD, Nelson EC, Montgomery GW, Martin NG. Zygosity diagnosis in the absence of genotypic data: an approach using latent class analysis. Twin Res 2003; 6: 22-26.

21 Ainsworth BE, Haskell WL, Whitt MC, Irwin ML, Swartz AM, Strath SJ, O'Brien WL, Bassett DR, Schmitz KH, Emplaincourt PO, Jacobs DR, Leon AS. Compendium of physical activities: an update of activity codes and MET intensities. Med Sci Sports Exerc 2000; 32: S498-S504.

22 Vastagh I, Horvath T, Garamvölgyi Z, Rosta K, Folyovich A, Rigó J Jr., Kollai M, Bereczki $D$, Somogyi A. Preserved structural and functional characteristics of common carotid artery in properly treated normoglycemic women with gestational diabetes mellitus. Acta Physiol Hung 2011; 98: 294-304.

23 Laurent S, Cockcroft JR, Van Bortel LM, Boutouyrie P, Giannattasio C, Hayoz D, Pannier B, Vlachopoulos C, Wilkinson IB, Struijker-Boudier Hon behalf of the European Network for Non-invasive Investigation of Large Arteries. Expert consensus document on arterial stiffness: methodological issues and clinical applications. Eur Heart J 2006; 27: 2588-2605.

24 Van Bortel LM, Duprez D, Starmans-Kool MJ, Safar ME, Giannattasio C, Cockcroft JR, Kaiser DR, Thuillez C. Clinical applications of arterial stiffness, Task Force III: recommendations for user procedures. Am J Hypertens 2002; 15: 445-452.

25 Asparouhov T. Sampling weights in latent variable modeling. Struct Equ Modeling 2005; 12: 411-434.

26 Agresti A. Categorical Data Analysis (2nd edn.Wiley-Interscience, New York, NY, 2002.

27 Neale M, Cardon LR. Methodology For Genetic Studies Of Twins And Families Vol. 67. Springer, 1992.

28 Bollen KA, Stine RA. Bootstrapping goodness-of-fit measures in structural equation models. Sociol Methods Res 1992; 21: 205-229.

29 Muthén LK, Muthén BO. Mplus. User's Guide 2007, pp. 233-287.

30 Pase MP, Grima NA, Sarris J. The effects of dietary and nutrient interventions on arterial stiffness: a systematic review. Am J Clin Nutr 2011; 93: 446-454.

31 Jatoi NA, Jerrard-Dunne P, Feely J, Mahmud A. Impact of smoking and smoking cessation on arterial stiffness and aortic wave reflection in hypertension. Hypertension 2007; 49: 981-985.

32 Pucci G, Battista F, Schillaci G. Aerobic physical exercise and arterial de-stiffening: a recipe for vascular rejuvenation? Hypertens Res 2012; 35: 964-966. 